University of Nebraska - Lincoln

DigitalCommons@University of Nebraska - Lincoln

Publications of the University of Nebraska

Public Policy Center

Public Policy Center, University of Nebraska

$2-8-2020$

\title{
A Review of Web-Based Special Education Resources Provided to Parents by State Departments of Education
}

Jennifer Farley

University of Nebraska-Lincoln, jfarley3@unl.edu

Jacqueline N. Huscroft-D'Angelo

University of Nebraska-Lincoln, jhuscrof@yahoo.com

Alexandra L. Trout

University of Nebraska - Lincoln, alex.trout@unl.edu

Kristin Duppong-Hurley

University of Nebraska-Lincoln, kristin.hurley@unl.edu

Follow this and additional works at: https://digitalcommons.unl.edu/publicpolicypublications

Part of the Other Public Affairs, Public Policy and Public Administration Commons, Other Social and Behavioral Sciences Commons, Public Affairs Commons, Public Policy Commons, and the Social Policy Commons

Farley, Jennifer; Huscroft-D'Angelo, Jacqueline N.; Trout, Alexandra L.; and Duppong-Hurley, Kristin, "A Review of Web-Based Special Education Resources Provided to Parents by State Departments of Education" (2020). Publications of the University of Nebraska Public Policy Center. 198.

https://digitalcommons.unl.edu/publicpolicypublications/198

This Article is brought to you for free and open access by the Public Policy Center, University of Nebraska at DigitalCommons@University of Nebraska - Lincoln. It has been accepted for inclusion in Publications of the University of Nebraska Public Policy Center by an authorized administrator of DigitalCommons@University of Nebraska - Lincoln. 


\title{
A Review of Web-Based Special Education Resources Provided to Parents by State Departments of Education
}

\author{
Jennifer Farley, PhD, Jacqueline Huscroft-D’Angelo, PhD, \\ Alexandra L. Trout, PhD, and Kristin Duppong Hurley, PhD
}

\author{
University of Nebraska-Lincoln, USA \\ Corresponding author - Jennifer Farley, University of Nebraska-Lincoln, \\ 212 Barkley Memorial Union, Lincoln, NE 68583, USA. \\ Email: jfarley3@unl.edu
}

\begin{abstract}
Parents who are knowledgeable of special education are more likely to engage in their child's education. Parents seek information about special education from a number of sources including State Departments of Education (SDEs). However, little is known about the web-based special education resources SDEs provide to parents. We sought to address this gap by conducting a comprehensive review of SDE websites and special education resources. Two-thirds of SDEs provided special education resources in a parent-designated section of their website. The number of resources provided varied greatly by state and the majority of resources were specific to conflict/dispute resolution. Future research should explore how information provided can best support increased parental knowledge to improve parental engagement. Limitations and implications are discussed.
\end{abstract}

Keywords: state agencies, parents, informational resources

Published in The Journal of Special Education 2020, Vol. 54(3) 180-190

DOI: $10.1177 / 0022466920903114$

Copyright (C) 2020 Hammill Institute on Disabilities; published by SAGE Publications. Used by permission. 
pecial education processes and services exist to ensure that all $\checkmark$ students have access to a Free and Appropriate Public Education (FAPE; Individuals With Disabilities Education Act [IDEA], 2004). Yet, the implementation of these processes and services are complex. Federal, state, and local policies and procedures guide the multifaceted process of identifying, evaluating, and serving children in special education programs. These programs must be unique and tailored to meet the diverse needs of students receiving special education. To accomplish these goals, many stakeholders may be involved throughout the process such as the child's parent(s), special education teacher, general education teacher, school psychologist, community advocate, specialist(s) (e.g., speech-language pathologist, occupational therapist), special education administrator, and the child (depending on age; IDEA, 2004). However, the student's parent is a unique and important stakeholder among the group; the parent has the most detailed and longitudinal data on the child, is the most likely person to be supporting and advocating for the child in the future, and is in the best position to bring about change in the child's life. As such, parents may be recognized as the best advocate for their child; as the primary advocate of their child, the possession of special education knowledge and skills is a beneficial asset (Burke et al., 2016). This includes knowledge of federal guidelines, evaluation, Individualized Education Plans, placement, services and accommodations, as well as skills related to partnering with schools (Burke et al., 2016).

Parent engagement in special education has been described as both "key" and "critical" for student success (Burke, 2012; Goldman \& Burke, 2017; Stoner et al., 2005). According to Spann et al. (2003), "parent participation leads to a host of positive outcomes for children with special needs" (p. 228). These outcomes may include better educational experiences, higher rates of homework completion, better grades, and fewer absences (Bryan \& Burstein, 2004; Poponi, 2009; Stoner et al., 2005). Parents may also be motivated to participate in special education to ensure their child receives needed services (Burke, 2012).

The importance of parental involvement in special education is emphasized in the IDEA (2004; Burke, 2013). This federal statute incorporates parents in multiple ways, including provisions for communication between parents and the school, inclusion of parents in 
student-level decision making and planning, granting parents access to information, and involving parents in systems-level decision making and planning (IDEA, 2004). When providing special education services to students, IDEA (2004) requires local education agencies (LEAs) to partner with parents, from initial parental consent for evaluation for special education services through placement and service provision. LEAs are required to (a) include parents as a member of their child's Individualized Education Program (IEP) team and schedule meetings so that parents can attend in-person or through alternative means (e.g., phone, video conference); (b) involve parents in determining educational placements, setting goals, and assessing progress; and (c) provide parents with specific information regarding their legal rights (i.e., procedural safeguards notice) and the identification, evaluation, and placement of their child (i.e., prior written notice; IDEA, 2004).

Overall, IDEA (2004) emphasizes the importance of parent engagement by positioning parents alongside experts, such as teachers and school personnel, in determining and implementing special education services. Parents are more likely to exhibit higher levels of engagement and partner with schools when they have knowledge and understanding of special education processes and services (Goldman \& Burke, 2017). Research indicates that parents who are not knowledgeable of school and legal policies, procedures, and terminology are less likely to become involved in the special education process (Burke, 2012; Fish, 2006, 2008). Alternatively, knowledge about special education helps parents to acquire necessary services for their child from the school (Fish, 2006; Lytle \& Bordin, 2001). Furthermore, understanding of parental rights in special education helps to form more equal partnerships between parents and school staff (Burke, 2012). Therefore, it is important that parents are supported to increase their knowledge and understanding of special education.

Parents are provided and may seek out information from a variety of sources to increase their understanding of special education. Schools are a primary source of information for parents, and research indicates the timely provision of information or training by schools increases parental knowledge and participation (Al-Hassan \& Gardner, 2002; Fish, 2008; Lo, 2008). Likewise, parents may also seek out information on their own through social networks and advocacy groups, Parent Training and Information Centers (PTI), State Departments of 
Education (SDEs), the internet, printed materials, seminars, trainings, and support groups (Burke, 2012; Family Empowerment and Disability Council, 2012; Leiter \& Krauss, 2004; Stoner et al., 2005). It is important for the information to be current, relevant, and reliable and that parents can easily assess the validity of the information (Family Empowerment and Disability Council, 2012).

Efforts to find reliable information may lead parents to search for web-based resources developed by federal and state agencies including Parent Information and Training Centers, national nonprofit organizations, SDEs, and school district websites. When considering both national and local organizations that provide web-based information to parents, SDEs emerge as unique organizations given their role, expertise, and credibility in special education policy creation and implementation. First, SDEs are uniquely situated between federal policy (IDEA) which involves parents and the LEAs that seek to engage them. From this position, SDEs provide policy and guidance for the administration of special education across each state. This includes the provision of funding to school districts, creation of administrative protocols, and the authority to decide how to address special education in the state using guidance from both federal and state policy (IDEA, 2004). SDEs also support both parents and school districts in recognizing parental and student rights, providing procedures for filing grievances, and resolving disputes (IDEA, 2004). Moreover, SDEs serve as a resource for LEAs, providing state-specific information and procedures for implementing special education services and much needed information and support to parents navigating the special education system.

It is evident that SDEs play a key role in the implementation of special education supports and services across the United States. SDEs may also act to increase parental knowledge of and engagement in special education by distributing informational resources through the SDE website. Yet, it remains unclear how SDEs disseminate information, including the quantity, content, and delivery of resources to parents, educators, and the public in general. The purpose of this study was to identify and examine the information provided to parents by state SDE websites to increase parent knowledge, understanding, and navigation of special education services and systems. Specifically, this study addresses the following research questions: (a) What 
information do SDE websites provide to parents regarding special education? and (b) In what manner (i.e., delivery method, language) do states provide this information to parents?

\section{Method}

We examined special education resources provided through SDE websites accessible to site users, including parents (e.g., available to the general public and not in password-secure SDE websites). Data were collected in two phases. Phase 1 included establishing the scope of the project, operationally defining inclusion criteria, creating a protocol for identification of SDE special education websites, an initial search of website resources, and determining final eligibility of all identified resources. Phase 2 focused on the detailed review of each identified resource and collecting data specific to variables of interest.

\section{Phase 1-Search Scope and Resource Inclusion}

Project scope. A systematic process was developed to review each SDE website for all 50 states and the Office of the State Superintendent of Education (OSSE) in Washington, D.C., which allowed for documenting variables of interest for each resource (see Phase 2). The review of SDE websites was completed between September 12, 2017 and January 3, 2018. Many SDE websites included links to resources provided by agencies external to the SDE, such as the U.S. Department of Education or community-based family advocacy organizations. Given the purpose of this study was to understand informational resources available to parents through SDE websites, resources provided on the websites of external agencies fell outside the scope of this study and were not included in the detailed review (see Phase 2). However, the links and states which provided each link were tracked. In addition, it was noted when the external links provided by SDEs were to PTI and/or Community Parent Resource Centers (CPRC) as these centers are a federally supported means of providing information about special education to parents and therefore central to the research questions. 


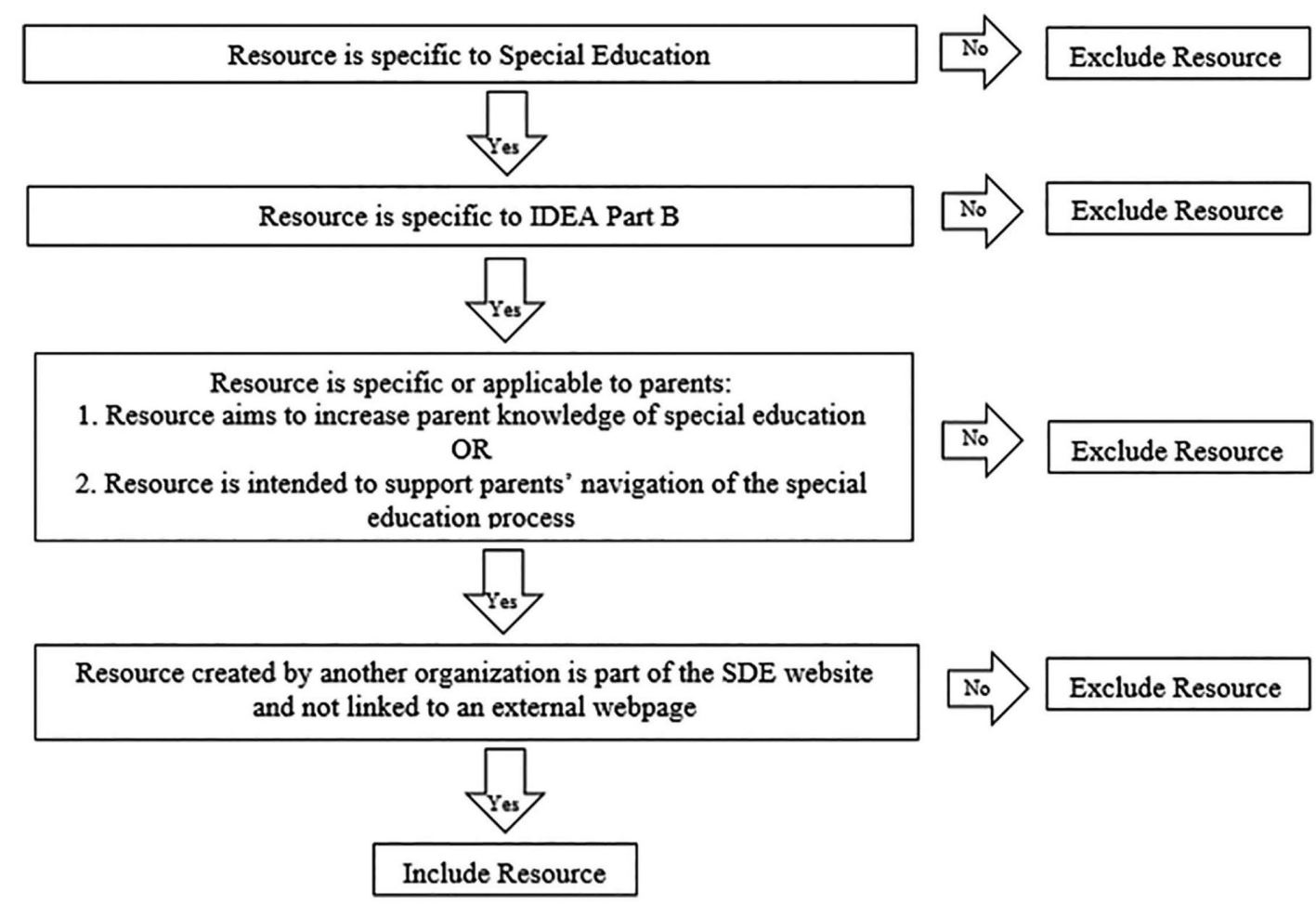

Figure 1. Criteria for inclusion of resources. Note. SDE = State Departments of Education; IDEA = Individuals With Disabilities Education Act.

Defining initial inclusion criteria. Inclusion criteria were defined for resources and content topics (e.g., parental rights, IEP, evaluation). Resources were defined as any source of information (e.g., PDF documents, pamphlets, presentations, videos) that may increase parents' knowledge and understanding of special education. Resources included broad topics (e.g., general parent's guide to special education, school services, Frequently Asked Questions [FAQs]) and more specific topics (e.g., autism, least restrictive learning environment, discipline). While some resources were created specifically for parents, this review included any resource on the SDE website which may support parents' understanding, knowledge, and navigation of special education, regardless of intended audience. Resources were included according to the criteria detailed in Figure 1.

SDE website search protocol. An initial search protocol was developed to ensure that the location and navigation of each SDE website 


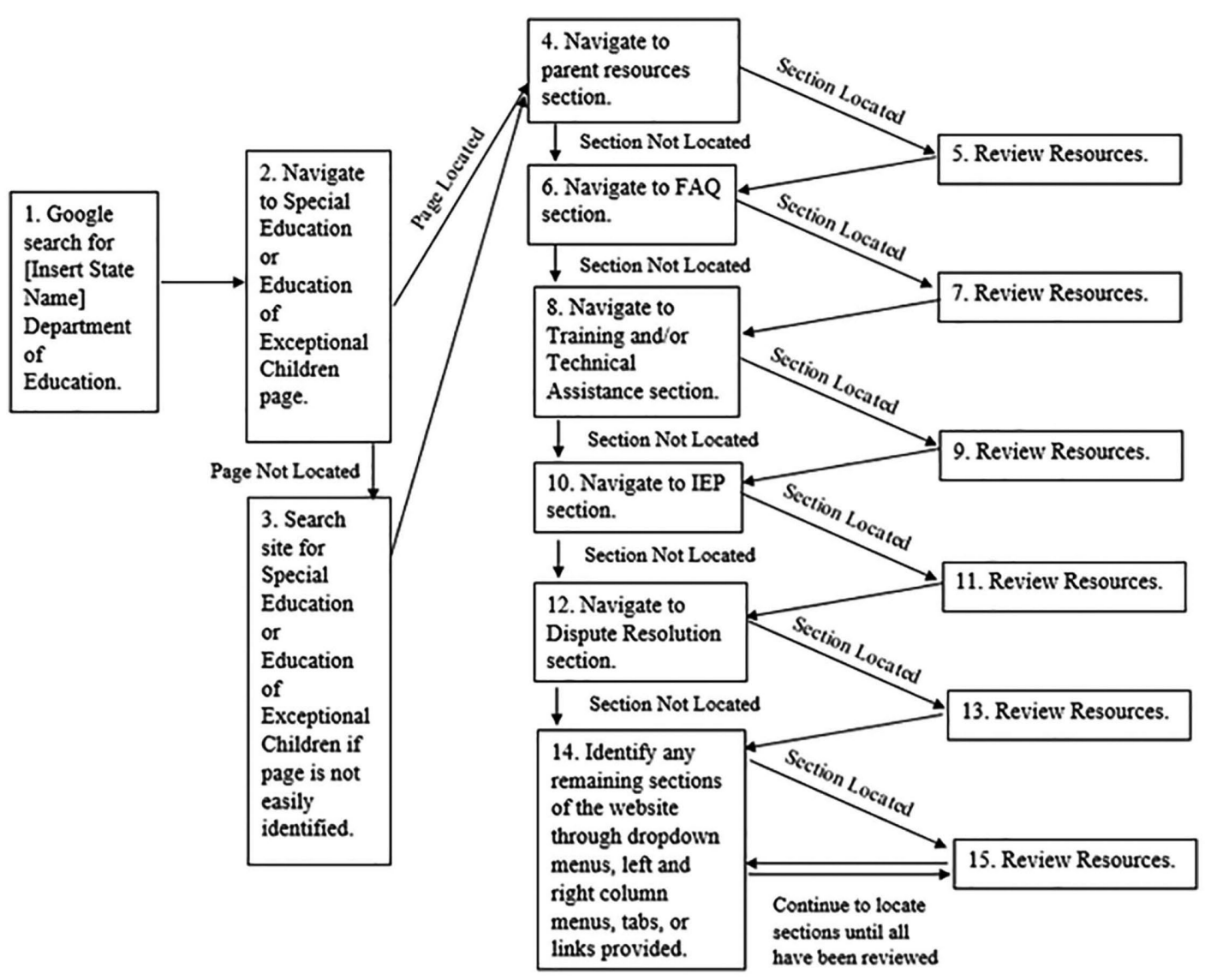

Figure 2. Sequential steps to search SDE websites and identify resources. Note. SDE $=$ State Departments of Education; FAQ = Frequently Asked Question; IEP = Individual Education Program.

was completed in the same order (see Figure 2). The outlined steps, detailed in Figure 2, were completed sequentially on the special education webpage(s) of each state website. All resources and embedded links to additional webpages were reviewed. After the resources and embedded links were reviewed for a given page, the search advanced to the next step.

The sequence of steps first allowed for a review of specific sections of each webpage, where parent resources were likely to be located. If a specific section of the website did not exist (e.g., Steps 3-6; parent resources, FAQ, IEP, dispute resolution), then the step was skipped and the next step was completed. The last step (Step 14) required the review of any additional sections of each special education webpage(s) to ensure all available resources were included. In instances where 
the same resource was located on multiple pages of the website, the resource was only reviewed once to determine inclusion.

Reliability of search protocol. Prior to independent reviews of SDE websites, reliability for the search protocol was assessed by the lead and second authors for the first six states following alphabetic order (i.e., Alabama-Colorado). Inter-rater agreement protocol required these reviewers to independently follow the search protocol and document each step (e.g., IP address of SDE website, IP address of special education page, names and links to resources) to identify resources. The decisions were then compared for each step and disagreements were discussed until the raters reached consensus. Inter-rater agreement, calculated as percentage of agreements for the total number of agreement opportunities, was 95\% across the 14 steps. Agreement was again measured after resources identified were reviewed (see Phase 2-Reliability of Resource Coding).

Final eligibility of resources. After the first six states were searched and inter-rater agreement was established, the team further refined inclusion criteria (see Figure 1). Initial criteria were expanded to include any resources that could increase parental knowledge and understanding of IDEA Part B. Resources did not have to be created specifically for parents, as opposed to educators, administrators, or community agencies, rather the resource simply had to support parents' understanding and knowledge of special education. For example, parents may have benefited from a guideline created for educators regarding the behavior and discipline of students receiving special education services, but parents would likely not benefit from a tutorial designed for school personnel to create a web-based IEP (e.g., EasyIEP).

SDE websites frequently included resources developed by external organizations or agencies. Resources provided by the state on the SDE website, rather than through a link to an external website, were included. Otherwise, they were considered external resources and tracked according to the number of states that provided a link to the external resource. In the case of the Kentucky Department of Education, the SDE website frequently highlighted a strong partnership with the University of Kentucky Human Development Institute, which 
included the provision of several parental resources on the Human Development Institute website. Because these resources were specific to Kentucky and provided by the SDE through the partnership, they were included; however, this was the only such instance of the inclusion of resources from an external website.

Finally, two types of resources were excluded. Forms, that served as a resource that should be printed and completed (e.g., blank forms to initiate the dispute resolution process), were excluded. These forms provided no actual information about special education services or processes, but instead sought to collect information from parents, thereby failed to meet inclusion criteria for the study. In addition, documents that provided excerpts from state/federal statutes or other legal codes with no further explanation or detail regarding the code were also excluded (e.g., state policy specific to restraint and seclusion, U.S. Department of Education procedural safeguards notice), as they simply repeated information available to parents elsewhere, did not attempt to support parent understanding, and thereby did not meet the study's criteria for inclusion.

\section{Phase 2-Coding of Included Variables}

Coding approach. The next step was to code all included resources. Resources were defined as a source of information or expertise in any form (e.g., print, video, learning module, training). In addition to the SDE name and date of the search, 20 additional characteristics of each resource were collected (see Table 1). Variables identified for inclusion in the data file were based on group-level variables identified by Goldman and Burke (2017). Given the project scope and research questions, additional variables were added to collect data about the format, delivery, and target population of the resource (additional details available upon request). Resources were excluded from the search when data collected indicated the resource was dated prior to 2004, the year of the most recent reauthorization of IDEA, or the link to the resource did not work. Variables of interest were not coded for the external resources, instead these resources were tracked according to the number of SDEs which provided the link to each resource. 
Table 1. Variables Collected for Each Identified Special Education Resource.

\begin{tabular}{|c|c|}
\hline Variable & Definition \\
\hline Agency name & Name of the SDE providing the resource. \\
\hline Name of resource & Name of the resource provided by the SDE. \\
\hline Working link & $\begin{array}{l}\text { Indicates whether the link to the resource provided on the } \\
\text { website was working and navigated to or opened the } \\
\text { intended resource. }\end{array}$ \\
\hline Part of parents' page & $\begin{array}{l}\text { Indicates whether the resource was located on a webpage } \\
\text { or section of the website specifically dedicated to } \\
\text { parent resources. }\end{array}$ \\
\hline Year & Year resource was created or updated. \\
\hline $\begin{array}{l}\text { Delivery mode } \\
\text { (select all that apply) }\end{array}$ & $\begin{array}{l}\text { The format in which the informational resource was } \\
\text { provided. Includes print, audio, video, website text, } \\
\text { in-person, and live video conference. }\end{array}$ \\
\hline $\begin{array}{l}\text { Core content/topics of } \\
\text { resource (choose all } \\
\text { that apply) }\end{array}$ & $\begin{array}{l}\text { Topic of the informational resource. Topics were specific } \\
\text { to steps in the Special Education process (U.S. } \\
\text { Department of Education, 20oo), including special } \\
\text { education, advocacy, assistive technology, autism, } \\
\text { behavior/discipline, child health, child skills, community } \\
\text { services, complaint/dispute resolution, condition, } \\
\text { diagnosis (special education category), evaluation, IEP, } \\
\text { interventions, law, least restrictive environment, parent } \\
\text { rights, referral, school services, self-care, strategies for } \\
\text { child skill building, terms, and transition. An "other" } \\
\text { option was also provided where additional topics could } \\
\text { be defined. }\end{array}$ \\
\hline Language (list all that apply) & The language(s) in which the resource was made available. \\
\hline Cost of resource & Any fee related to the use of the resource, in dollars. \\
\hline
\end{tabular}

Variable and value definitions are available upon request. SDE = State Departments of Education; IEP = Individual Education Program.

Reliability of resource coding. Initial reliability was obtained by reviewing the resources of six (11\%) SDE websites. All resources within the six websites were double-coded and assessed for reliability between the lead and second authors. Inter-rater agreement required each reviewer to independently code the resource records. The decisions were then compared for each variable related to the resource on the coding sheet. All disagreements were discussed, and final agreement was reached by consensus. Inter-rater agreement, calculated as percentage of agreements for the total number of agreement opportunities, was $87 \%$ across the six SDE websites. After high initial reliability was obtained, the remaining state resources were identified and 
recorded by one team member; however, random reliability checks were conducted on $23 \%$ of resources to ensure maintenance of at least $80 \%$ reliability.

\section{Data Collection and Analysis}

SDE websites were reviewed in alphabetical order according to state name. A MS Excel file was created to track and organize data collected for each identified resource and coded variables. For open ended variables, data were entered as text (e.g., SDE, resource name, length of resource). Other variables included multiple dichotomous response options (see Table 1; e.g., target population, delivery mode, topic, language). When variable data were unavailable (e.g., year resource was created), the corresponding cell was assigned a value to indicate missing.

In addition to the variables outlined in Table 1, the MS Excel File also included two additional worksheets to collect data for two separate variables. The first worksheet tracked whether each SDE website had a specific webpage or section of a webpage devoted to parent resources and included a link to the webpage. The second worksheet tracked external links or links to resources provided by other organizations including the federal government, universities, and national, state, and community agencies. The resources provided through these links were tracked according to the external agency which created the resource (e.g., U.S. Department of Education, Center for Appropriate Dispute Resolution in Special Education, Center for Parent Information and Resources), the name of the resource, and the names of the SDEs which provided a link to the resource.

Data were inspected, and results were summarized for each variable and value. Topics defined as other were coded and the categories Autism $(n=16 ; 2.24 \%)$ and Assistive Technology $(n=18 ; 2.52 \%)$ emerged, as they both contained more than $2 \%$ of all resources. Records and summaries were reviewed to ensure data entry was complete. Frequency distributions and measures of central tendency were calculated. Finally, descriptive statistics were used to compare states and resources across variables. 


\section{Results}

The review of 51 SDE websites identified 752 resources related to special education. Of these, nine were excluded because of publication dates prior to 2004. An additional 29 resources were excluded due to broken links which did not allow for the resource to be reviewed and coded. As a result, 714 resources were coded.

\section{Informational Resources}

The number of resources provided by each SDE ranged from 2 to 68, with a median of $10(M=14 ; S D=11.57$; See Figure 3$)$. Across the 714 resources, $341(47.8 \%)$ included the year the resource was created. Dates ranged from $2004(n=8)$ to $2018(n=1)$, with the greatest number of resources created or updated in $2017(n=85)$. When examining the location of resources provided on various webpages within the SDE website, 34 (66.7\%) of SDE special education webpages devoted a page, or section of a webpage, to resources for parents and families, identifiable by titles such as "Family Information," "Parents/Families," or "Parent Resources." The remaining 17 (33.3\%) states provided resources applicable to parents, but these were located within the special education webpages rather than in a section of the webpage specific to parents. The 34 states that provided a parent webpage or section also provided additional resources applicable to parents elsewhere on their webpage(s).

Topics. A diverse set of special education-related topics were included on SDE websites. Not all states provided resources related to the same topics. Resources provided by states covered between 3 and 17 topics, with an average of 9.7 topics $(S D=3.5)$. Just more than half of the resources $(51.3 \% ; n=366)$ were specific to one topic and 348 resources $(48.7 \%)$ included multiple topics. The greatest number of resources were about conflict and dispute resolution ( $n=216 ; 30.3 \%)$. Figure 4 illustrates the number of resources provided on each topic.

The 34 SDEs with parent webpages/sections provided informational resources on multiple topics within these parts of the webpage. More than half of these 34 states maintained parent webpages which included resources specific to IEPs ( $n=22 ; 64.7 \%)$, complaint/ 


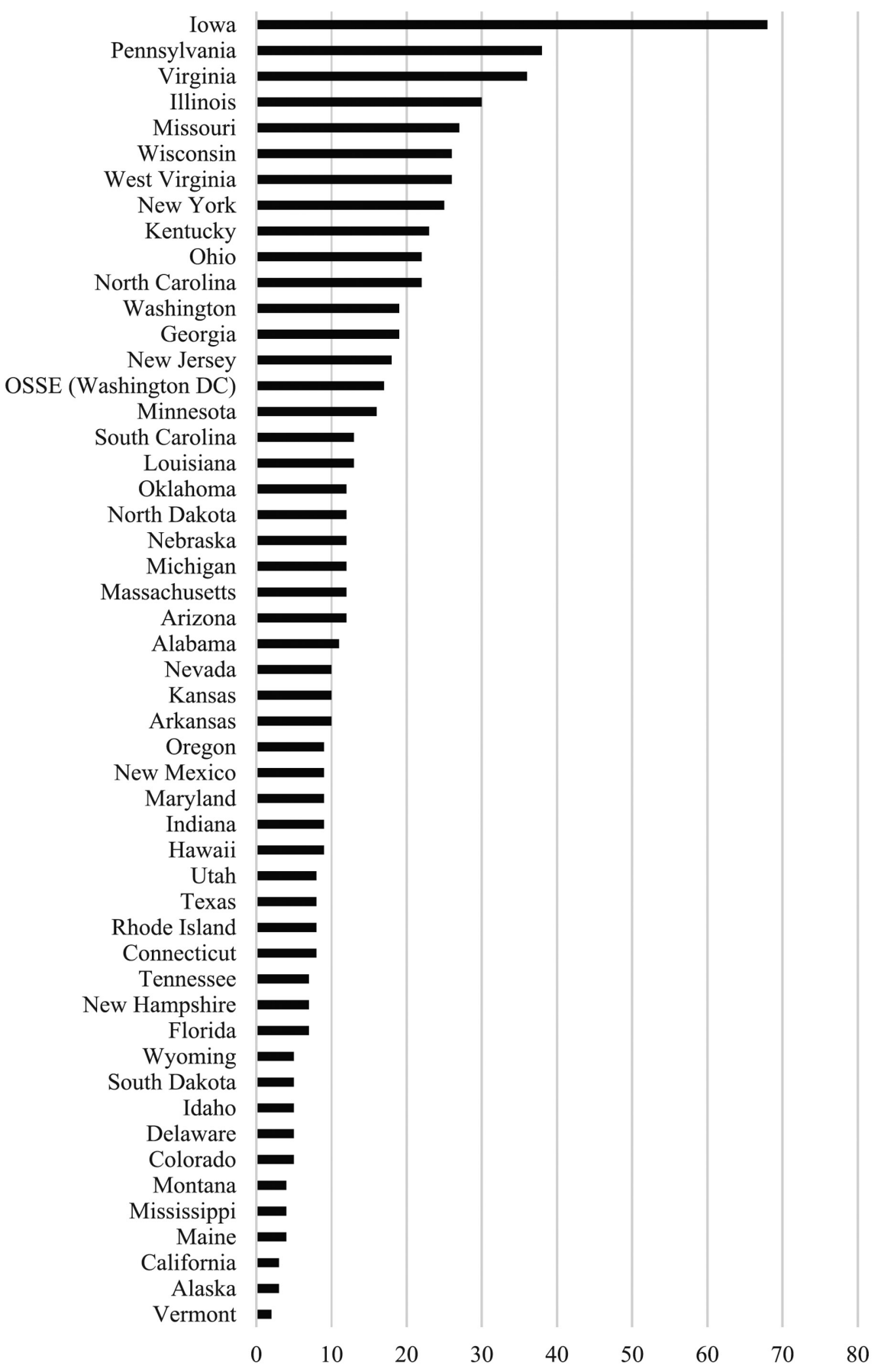

Figure 3. Total number of resources provided by state.

dispute resolution ( $n=19 ; 55.9 \%)$, evaluation ( $n=18 ; 52.9 \%)$, and parent rights $(n=17 ; 50.0 \%)$. 
External resources. SDE websites frequently included links to resources which were provided by and located on the websites of external agencies. External agencies were varied, including federal agencies, nonprofit organizations, and universities. A total of 666 ( $M=$ 17.9; $S D=17.9$; range $=1-92$ ) links to unique external resources were provided on SDE websites. The most frequently provided link was to the Center for Appropriate Dispute Resolution in Special Education (www.cadreworks.org), included on 14 SDE websites. Links to the National Technical Assistance Center on Transition, National Center on Accessible Educational Materials, and American Printing House for the Blind, Inc. were each provided by seven SDEs.

Parent centers. While links to external resources were primarily examined by state and organization, as each state has Office of Special Education Programs (OSEP) federal dollars to support their own state PTI and/or CPRC, we also examined these resources collectively. This did not include local community agencies or nonprofits not receiving OSEP funds for support. Twenty-seven states provided 44 links to resources provided by $38 \mathrm{PTI} / \mathrm{CPRC}$ agencies, which represented $7 \%$ of all links to external agencies $(n=666)$. The total number of links per state ranged from one to three. The PACER Center (Minnesota PTI) was the only PTI referenced by more than one state and was referenced by four SDEs. Most frequently, links provided routed users to the homepage of the PTI/CPRC organization ( $n=23)$; however, links also provided information on activities, trainings, and groups $(n=7)$ as well as content-specific resources $(n=7)$.

\section{Provision of Informational Resources}

Delivery mode. Resources provided for parents on SDE websites were available in several formats including print (66\%), website text (25\%), video (8\%), in-person conferences (1\%), and audio (0.4\%). Print resources ( $n=475 ; 66 \%)$, or those resources which were available through a website link to a document that could be printed (e.g., PDF, MS Word document), were the most frequently provided by states. The second most common mode of delivery was text embedded within the SDE website $(n=181 ; 25 \%)$. These text-embedded resources differed from print resources in that they were incorporated 
Number of Resources and States Providing Resources by Topic ${ }^{\mathrm{a}}$

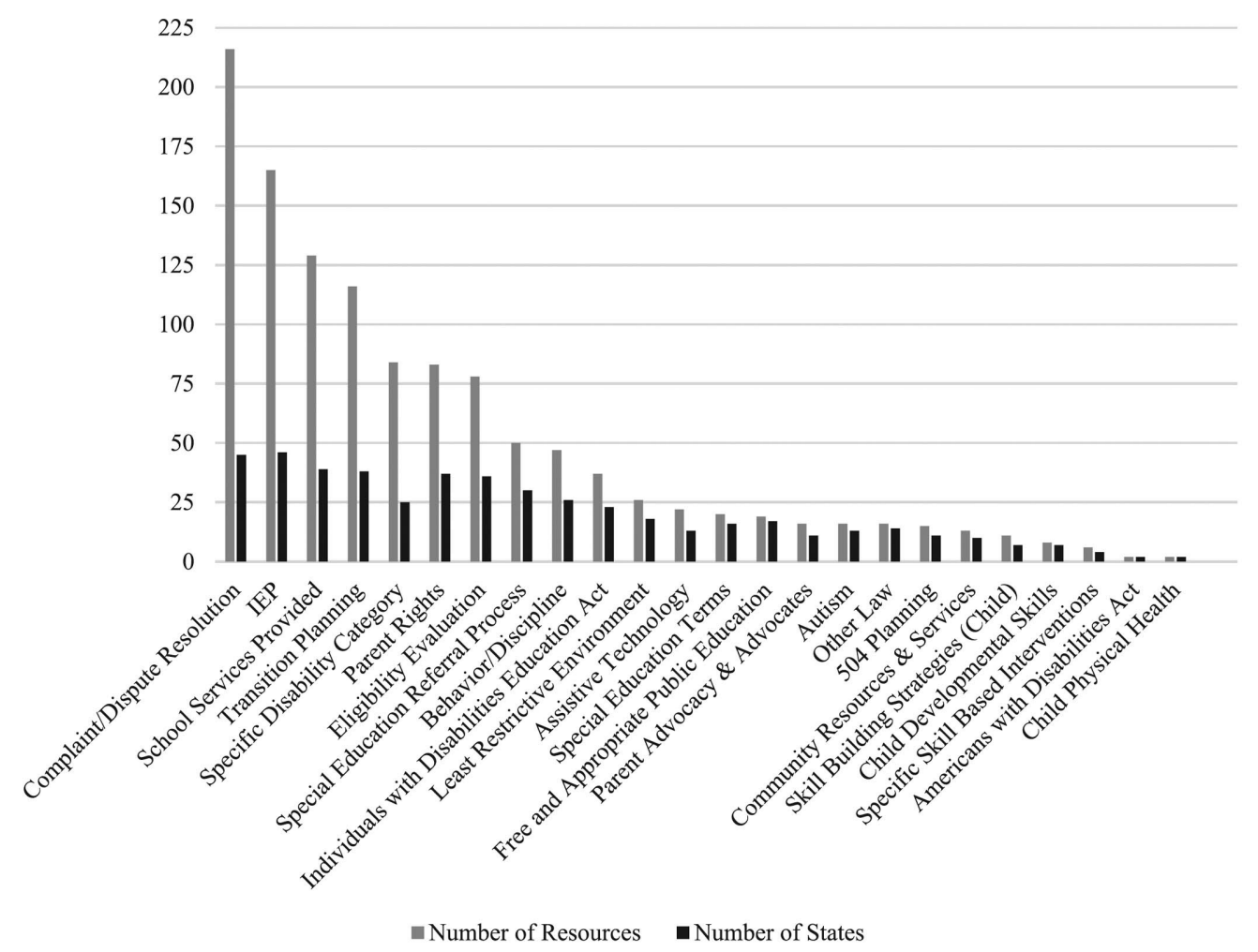

Figure 4. Number of resources and states providing resources by topic. a. Categories are not exclusive; resources with multiple topics are counted each applicable topic category.

into the webpage, did not provide links to materials created in other software programs, and could only be printed if the webpage was printed. In-person informational resources included conferences or training opportunities. Nine states $(17.7 \%)$ provided resources only in print, while the remaining states provided resources in multiple delivery modes (i.e., video, audio, in-person).

Accessibility. Thirty-one states (6o.8\%) provided at least one resource in a language other than English. For these states, the number of resources available in more than one language ranged from one to seven $(M=2.42, S D=1.5)$. This accounted for between $1 \%$ and $50 \%$ of the total resources provided by each of these states. The majority of resources ( $n=639 ; 89.5 \%)$ were only available in English. Of the 
75 resources available in other languages, 100\% were available in Spanish and 33 (44.0\%) were available in three or more languages. Resources available in languages other than English were most likely to be specific to the topics of complaint and dispute resolution ( $n=$ $43 ; 57.3 \%)$, parent rights $(n=24 ; 32.0 \%)$, and IEPs $(n=20 ; 26.7 \%)$.

\section{Discussion}

Prior to becoming engaged in special education, parents must be knowledgeable about the processes and services available to their child. This knowledge and understanding of special education may be supported by informational resources, many of which are provided on SDE websites. Parents may see such resources as reputable and of high quality given the SDE's role in special education provision within any given state. Yet, little is known about the resources made available to parents by SDEs. This study is the first to explore the extent to which SDEs provide information about special education, with respect to quantity, content, and delivery.

\section{Informational Resources}

While generally SDEs provided a large number of resources to parents, states providing very few resources, such as the six states that provided less than five, may not provide enough information to meet the needs of parents. This may result in parents' limited understanding, disadvantage in interactions with educators (Fish, 2008), dissatisfaction with special education (Stoner et al., 2005), and lack of participation in the special education process (Burke, 2012; Fish, 2006; Fitzgerald \& Watkins, 2006). Parents' understanding of special education may also be influenced by dated information, yet publication dates were not available for $373(52 \%)$ resources. While the lack of a publication date certainly does not indicate resources are outdated, it is possible that some resources provided by SDEs may no longer be current or may include information that is no longer relevant.

Perhaps more significant than the quantity of informational resources provided by states was the organization of these resources on SDE websites. Surprisingly, a third of states did not designate any 
portion of their special education webpage(s) to parent-specific content regarding special education services. Moreover, the 34 states with pages or sections of their special education website devoted to parents did not feature all parent resources on those pages, which would require parents to continue searching the SDE website for needed information, beyond the parent special education page. Such extensive searching may make it difficult for parents to find the information they need to become knowledgeable about special education, thus reducing their likelihood to engage and partner with schools in the special education process (Goldman \& Burke, 2017).

Topics. While it was not the intent of this study to conduct a comprehensive analysis of the content of informational resources, each was coded by topic to gain an initial understanding of content. When reviewing the topics of web content across all SDEs, resources were most frequently provided specific to dispute resolution and IEPs. While dispute resolution is an important topic, and SDEs should be commended for providing resources to build knowledge on this topic, this information is applicable to very few families. During 2015-2016, 5,319 special education complaints were filed across all 50 states and the District of Columbia (U.S. Department of Education, 2017a, 2017b), representing approximately .08\% of students receiving special education services. While provision of so many resources on this topic may reflect the role of SDEs in dispute resolution, as outlined in IDEA (2004), it is surprising that resources specific to IEPs, which are relevant to all parents of children receiving special education services, are the second most frequently provided resource, instead of the first. It is essential that parents are knowledgeable about the IEP process, especially given their role on the IEP team as an advocate for their child (Family Empowerment and Disability Council, 2012; IDEA, 2004). Furthermore, parent advocacy skills are needed for active engagement in special education (Burke et al., 2016), yet only 16 total resources (.14\%), provided by 11 states (21.6\%), were related to advocacy. Continued or increased provision of resources with content relevant to all families may further support parental knowledge of special education and increase engagement (Goldman \& Burke, 2017). This in turn may prevent instances of dispute resolution. 
External resources. It was notable how often parents were referred to external resources for information. Attention to the organization of external resources may also improve parents' success in obtaining the information they need. For example, it is more helpful to include a link to a specific resource within a website rather than just linking to the home page of a website and requiring the parent to search for the relevant information from the home page.

External resources most frequently connected users to PTI or CPRC websites. Given that these agencies exist to support, educate, and engage parents of students in special education, it was surprising to find they made up such a small percentage external resources (7\%) and are provided by only about half of all SDEs (53\%). This raises questions regarding how SDEs currently partner with PTIs to provide information to parents of students in special education.

\section{Provision of Informational Resources}

In addition to exploring and analyzing what special education information was provided through SDE websites, this study sought to understand how informational resources were provided. It is not surprising that the majority of information was provided in print, given that websites are primarily text-driven in nature and videos can be expensive and time-consuming to produce. However, given the potential for reading and comprehension barriers (Fitzgerald \& Watkins, 2006), provision of information in multiple formats would ensure all parents were able to access resources.

Accessibility. While approximately 10\% of students receiving special education services are English Language Learners (ELLs; U.S. Department of Education, 2017c), results indicate that the majority of the materials provided on SDE websites were only available in English and that materials available in languages other than English were frequently associated with procedural safeguards. While one expects that parents are receiving this information from school districts in their native language, as required by IDEA (2004), it could be beneficial for SDEs to consider translating resources that would further support increased parental knowledge of special education. This would be especially meaningful for parents unfamiliar with both the general 
and special education systems in the United States while also experiencing a language barrier (Al-Hassan \& Gardner, 2002). While some internet browsers allow users to customize websites to their own native language, thus reducing barriers related to accessing web content, such technology does not necessarily extend to the informational resources provided as printed documents (e.g., PDF, MS Word Documents) or video/audio formats.

\section{Implications}

This study revealed several areas for further research. The first priority for future research must be to better understand how SDEs view their role in providing special education information to parents and, specifically, how the provision of such resources aligns with the SDE's role and mission. This includes understanding SDE choices in how resources are provided, including designation of a webpage or section of the webpage specifically for parents, and overall organization of resources for easy access by parents. Better understanding of how SDEs view their role in the provision of information may provide insight into why some states provide considerable resources for parents and others do not seem to identify parents as a key website user. This research should also address how SDEs use external resources to support parents' access to information about special education including the development of a better understanding of how SDEs partner with PTIs and CPRCs to inform parents.

The second priority of further research is related to the quantity, quality, and content of informational resources provided by SDEs, both on SDE websites and through external links. Focused attention on the quality and comprehensiveness of existing resources may help to better understand how SDEs determine the quantity of resources to provide. Additional research is required to determine what special education content parents most need, the order in which such information is presented, and how to make navigation of such information easy and straightforward. SDE website content devoted to the general processes and services of special education may benefit more parents than the provision of parental rights materials (required by IDEA). Research on how to best improve the availability of these more general special education resources may help parents to access the information they 
need to prevent disagreements between parents and school districts from escalating into the formal dispute resolution process. Expansion and replication of existing pilot studies on parental access and use of special education resources may identify parent and school preferences for content and delivery of informational resources (e.g., Huscroft-D'Angelo et al., 2018). Ultimately, such research could inform the development of best practices for providing information to build parent knowledge of special education. Implementation of such practices by SDEs would then allow for more equitable access to informational resources by parents, independent of their geographic location.

Finally, the third priority of additional research is specific to how parents use the SDE website and the accessibility of informational resources provided by SDEs. Additional research is necessary to determine the topics on which parents seek information and parents' preferred formats for receiving information (i.e., video, audio, printed). However, integral to this is a better understanding of how easy or difficult it is for parents to navigate the SDE website to find needed information, including whether parents encounter barriers to accessing special education information provided on SDE websites due to the language and readability of the resources provided. Equal access to information about special education processes and services will ensure that families are supported to become an informed, and ultimately engaged, partner in special education.

\section{Limitations}

Several study limitations should be noted. First, the study was time limited as it was conducted between September, 2017, and January, 2018, providing only a snapshot of the resources provided by SDEs, which are continually subject to change. Second, while the study reports a number of variables related to the resources provided by SDEs, these factors do not provide a comprehensive examination of content or an overall measure of the quality of information provided. Further research would allow for the assessment of quality of resources and may include measurement of variables such as readability, author expertise, and context. Third, additional studies would also allow for better understanding of how SDEs determine what information to provide on the SDE website and how stakeholder audiences are 
supported. Fourth, this review was limited to the resources provided on SDE websites, with limited information collected regarding external resources provided. Future research should take into account both the content and manner in which external websites provide information to parents. Finally, additional research is needed to provide a better understanding of how parents use SDE informational resources, how they identify and search for information they need, how they prefer to access information, and ultimately how these resources inform their understanding of special education services.

This is the first known study to analyze the informational resources provided on SDE websites specific to special education. As a result, it reveals just the beginning of what needs to be known regarding how parents' understanding of special education is informed by resources provided by SDE websites. The primary finding is that SDEs vary considerably in terms of the quantity, content, organization, and delivery of special education resources to parents, which may lead to differences in parents' success obtaining needed information about special education according to their state of residence. Future research is needed to assess the quality of informational resources provided by SDEs, explore how resources are selected for dissemination on SDE websites, and identify any barriers parents with diverse learning and language needs may experience when accessing special education resources. Ultimately, a better understanding of how parents use SDEs to find information about special education, and how such resources inform their engagement in special education, is critical to the context of this and future studies.

Authors' note The opinions expressed are those of the authors and do not necessarily represent views of the Institute or the U.S. Department of Education.

Declaration of Conflicting Interests The authors have declared no potential conflicts of interest with respect to the research, authorship, and publication of this article.

Funding The preparation of this manuscript was supported by the Institute of Education Sciences (IES), U.S. Department of Education, through Grant no. R324B160033 to the University of Nebraska-Lincoln. 


\section{References}

Al-Hassan, S., \& Gardner, R. (2002). Involving immigrant parents of students with disabilities in the educational process. Teaching Exceptional Children, 38(5), $52-58$.

Bryan, T., \& Burstein, K. (2004). Improving homework completion and academic performance: Lessons from special education. Theory Into Practice, 43, 213-219.

Burke, M. (2012). Examining family involvement in regular and special education: Lessons to be learned for both sides. International Review of Research in Developmental Disabilities, 43, 187-218.

Burke, M. (2013). Improving parental involvement: Training special education advocates. Journal of Disability Policy Studies, 23, 225-234.

Burke, M., Goldman, S., Hart, S., \& Hodapp, R. (2016). Evaluating the efficacy of a special education advocacy training program. Journal of Policy and Practice in Intellectual Disabilities, 13, 269-276.

Family Empowerment and Disability Council. (2012). The Individuals With Disabilities Education Act and parental participation [FEDC issue brief]. http:// www.efrconline.org/myadmin/files/fedc Parent Participation.pdf

Fish, W. (2006). Perceptions of parents of students with autism towards the IEP meeting: A case study of one family support group chapter. Education and Treatment of Children, 127, 56-68.

Fish, W. (2008). The IEP meeting: Perceptions of parents of students who receive special education services. Preventing School Failure, 53, 8-14.

Fitzgerald, J., \& Watkins, M. (2006). Parents' rights in special education: The readability of procedural safeguards. Exceptional Children, 72, 497-510.

Goldman, S., \& Burke, M. (2017). The effectiveness of interventions to increase parent involvement in special education: A systematic literature review and meta-analysis. Exceptionality, 25, 97-115.

Huscroft-D’Angelo, J., Farley, J., Trout, A., \& Duppong Hurley, K. (2018, October). Preparing parents to engage in the special education process: Knowledge, training and access to resources [Paper presentation]. Presentation at the Teacher Educators for Children with Behavior Disorders Conference, Tempe, AZ.

Individuals With Disabilities Education Act, 20 U.S.C. § 1400 (2004).

Leiter, V., \& Krauss, M. (2004). Claims, barriers, and satisfaction: Parents' requests for additional special education services. Journal of Disability Policy Studies, 15, 135-146.

Lo, L. (2008). Chinese families' level of participation and experiences in IEP meetings. Preventing School Failure, 53, 21-27.

Lytle, R., \& Bordin, J. (2001). Enhancing the IEP team. Teaching Exceptional Children, 33(5), 40-44.

Poponi, D. M. (2009). The relationship between student outcomes and parental involvement in multidisciplinary IEP team meetings [Doctoral dissertation]. https://digitalcommons.pcom.edu/psychology dissertations/116 
Spann, S., Kohler, F., \& Soenksen, D. (2003). Examining parents' involvement in and perceptions of special education services: An interview with families in a parent support group. Focus on Autism and Other Developmental Disabilities, $18,228-237$.

Stoner, J. B., Jones Bock, S., Thompson, J. R., Angell, M. E., Heyl, B. S., \& Crowley, E. P. (2005). Welcome to our world: Parent perceptions of interactions between parents of young children with ASD and education professionals. Focus on Autism and Other Developmental Disabilities, 20, 39-51.

U.S. Department of Education. (2000). A Guide to the Individualized Education Program. https://www2.ed.gov/parents/needs/speced/iepguide/index.html

U.S. Department of Education. (2017a). IDEA Part B child count and educational environments collection [EDFacts Data Ware house (EDW)]. http://www2. ed.gov/programs/osepidea/618-data/index.html

U.S. Department of Education. (2017b). IDEA Part B Dispute Resolution Survey [EDFacts Metadata and Process System (EMAPS)]. http://www2.ed.gov/ programs/osepidea/618-data/index.html

U.S. Department of Education. (2017c). State nonfiscal survey of public elementary and secondary education, 2008-og through 2015-16 [EDFacts Data Warehouse]. https://nces.ed.gov/programs/digest/d17/tables/dt17_204.27.asp 